Supplement of Clim. Past Discuss., 10, 2011-2053, 2014

http://www.clim-past-discuss.net/cpd-10-2011-2014/

doi:10.5194/cpd-10-2011-2014-supplement

(C) Author(s) 2014. CC Attribution 3.0 License.

(c) (i)

Supplement of

\title{
Radiative forcings for 28 potential Archean greenhouse gases
}

Correspondence to: B. Byrne (bbyrne@uvic.ca) 
Table 1. Atmospheric structure for 0.5 bar of $\mathrm{N}_{2}$

GAM Profile

\begin{tabular}{|c|c|c|c|}
\hline $\mathrm{P}(\mathrm{Pa})$ & $\mathrm{T}(\mathrm{K})$ & $\mathrm{H} 2 \mathrm{O}$ (mass fraction) & $\mathrm{N}_{2}$ (mass fraction) \\
\hline $5.0249 \times 10^{0}$ & $2.0300 \times 10^{2}$ & $7.8796 \times 10^{-6}$ & $9.9999 \times 10^{-1}$ \\
\hline $1.5075 \times 10^{1}$ & $2.0300 \times 10^{2}$ & $7.8796 \times 10^{-6}$ & $9.9999 \times 10^{-1}$ \\
\hline $2.0100 \times 10^{1}$ & $2.0300 \times 10^{2}$ & $7.8796 \times 10^{-6}$ & $9.9999 \times 10^{-1}$ \\
\hline $2.5124 \times 10^{1}$ & $2.0300 \times 10^{2}$ & $7.8796 \times 10^{-6}$ & $9.9999 \times 10^{-1}$ \\
\hline $3.5174 \times 10^{1}$ & $2.0300 \times 10^{2}$ & $7.8796 \times 10^{-6}$ & $9.9999 \times 10^{-1}$ \\
\hline $5.0249 \times 10^{1}$ & $2.0300 \times 10^{2}$ & $7.8796 \times 10^{-6}$ & $9.9999 \times 10^{-1}$ \\
\hline $1.0050 \times 10^{2}$ & $2.0300 \times 10^{2}$ & $7.8796 \times 10^{-6}$ & $9.9999 \times 10^{-1}$ \\
\hline $1.5075 \times 10^{2}$ & $2.0300 \times 10^{2}$ & $7.8796 \times 10^{-6}$ & $9.9999 \times 10^{-1}$ \\
\hline $2.0100 \times 10^{2}$ & $2.0300 \times 10^{2}$ & $7.8796 \times 10^{-6}$ & $9.9999 \times 10^{-1}$ \\
\hline $2.5124 \times 10^{2}$ & $2.0300 \times 10^{2}$ & $7.8796 \times 10^{-6}$ & $9.9999 \times 10^{-1}$ \\
\hline $3.5174 \times 10^{2}$ & $2.0300 \times 10^{2}$ & $7.8796 \times 10^{-6}$ & $9.9999 \times 10^{-1}$ \\
\hline $5.0249 \times 10^{2}$ & $2.0300 \times 10^{2}$ & $7.8796 \times 10^{-6}$ & $9.9999 \times 10^{-1}$ \\
\hline $1.0050 \times 10^{3}$ & $2.0300 \times 10^{2}$ & $7.8796 \times 10^{-6}$ & $9.9999 \times 10^{-1}$ \\
\hline $1.5075 \times 10^{3}$ & $2.0300 \times 10^{2}$ & $7.8796 \times 10^{-6}$ & $9.9999 \times 10^{-1}$ \\
\hline $2.0100 \times 10^{3}$ & $2.0300 \times 10^{2}$ & $7.8796 \times 10^{-6}$ & $9.9999 \times 10^{-1}$ \\
\hline $2.5124 \times 10^{3}$ & $2.0300 \times 10^{2}$ & $7.8796 \times 10^{-6}$ & $9.9999 \times 10^{-1}$ \\
\hline $3.5174 \times 10^{3}$ & $2.0300 \times 10^{2}$ & $7.8796 \times 10^{-6}$ & $9.9999 \times 10^{-1}$ \\
\hline $5.0249 \times 10^{3}$ & $2.0300 \times 10^{2}$ & $7.8796 \times 10^{-6}$ & $9.9999 \times 10^{-1}$ \\
\hline $7.5247 \times 10^{3}$ & $2.0300 \times 10^{2}$ & $7.8796 \times 10^{-6}$ & $9.9999 \times 10^{-1}$ \\
\hline $7.5373 \times 10^{3}$ & $2.0311 \times 10^{2}$ & $7.9180 \times 10^{-6}$ & $9.9999 \times 10^{-1}$ \\
\hline $1.0050 \times 10^{4}$ & $2.2072 \times 10^{2}$ & $7.1484 \times 10^{-5}$ & $9.9993 \times 10^{-1}$ \\
\hline $1.1017 \times 10^{4}$ & $2.2600 \times 10^{2}$ & $1.6833 \times 10^{-4}$ & $9.9983 \times 10^{-1}$ \\
\hline $1.2562 \times 10^{4}$ & $2.3443 \times 10^{2}$ & $3.4760 \times 10^{-4}$ & $9.9965 \times 10^{-1}$ \\
\hline $1.5075 \times 10^{4}$ & $2.4479 \times 10^{2}$ & $9.1247 \times 10^{-4}$ & $9.9909 \times 10^{-1}$ \\
\hline $1.7587 \times 10^{4}$ & $2.5254 \times 10^{2}$ & $1.6120 \times 10^{-3}$ & $9.9839 \times 10^{-1}$ \\
\hline $2.0100 \times 10^{4}$ & $2.5849 \times 10^{2}$ & $2.4013 \times 10^{-3}$ & $9.9760 \times 10^{-1}$ \\
\hline $2.2612 \times 10^{4}$ & $2.6324 \times 10^{2}$ & $3.1905 \times 10^{-3}$ & $9.9681 \times 10^{-1}$ \\
\hline $2.5016 \times 10^{4}$ & $2.6700 \times 10^{2}$ & $3.9457 \times 10^{-3}$ & $9.9605 \times 10^{-1}$ \\
\hline $2.5124 \times 10^{4}$ & $2.6717 \times 10^{2}$ & $3.9841 \times 10^{-3}$ & $9.9602 \times 10^{-1}$ \\
\hline $2.7637 \times 10^{4}$ & $2.7051 \times 10^{2}$ & $5.0553 \times 10^{-3}$ & $9.9494 \times 10^{-1}$ \\
\hline $3.0149 \times 10^{4}$ & $2.7340 \times 10^{2}$ & $6.3234 \times 10^{-3}$ & $9.9368 \times 10^{-1}$ \\
\hline $3.2662 \times 10^{4}$ & $2.7598 \times 10^{2}$ & $7.2918 \times 10^{-3}$ & $9.9271 \times 10^{-1}$ \\
\hline $3.5174 \times 10^{4}$ & $2.7831 \times 10^{2}$ & $8.2867 \times 10^{-3}$ & $9.9171 \times 10^{-1}$ \\
\hline $3.6430 \times 10^{4}$ & $2.7940 \times 10^{2}$ & $8.8656 \times 10^{-3}$ & $9.9113 \times 10^{-1}$ \\
\hline $3.7154 \times 10^{4}$ & $2.8000 \times 10^{2}$ & $9.1997 \times 10^{-3}$ & $9.9080 \times 10^{-1}$ \\
\hline $3.7687 \times 10^{4}$ & $2.8044 \times 10^{2}$ & $9.4394 \times 10^{-3}$ & $9.9056 \times 10^{-1}$ \\
\hline $3.8943 \times 10^{4}$ & $2.8144 \times 10^{2}$ & $9.9618 \times 10^{-3}$ & $9.9004 \times 10^{-1}$ \\
\hline $4.0199 \times 10^{4}$ & $2.8240 \times 10^{2}$ & $1.0487 \times 10^{-2}$ & $9.8951 \times 10^{-1}$ \\
\hline $4.1455 \times 10^{4}$ & $2.8333 \times 10^{2}$ & $1.1196 \times 10^{-2}$ & $9.8880 \times 10^{-1}$ \\
\hline $4.2711 \times 10^{4}$ & $2.8422 \times 10^{2}$ & $1.2286 \times 10^{-2}$ & $9.8771 \times 10^{-1}$ \\
\hline $4.3968 \times 10^{4}$ & $2.8508 \times 10^{2}$ & $1.3786 \times 10^{-2}$ & $9.8621 \times 10^{-1}$ \\
\hline $4.5224 \times 10^{4}$ & $2.8592 \times 10^{2}$ & $1.5477 \times 10^{-2}$ & $9.8452 \times 10^{-1}$ \\
\hline $4.6480 \times 10^{4}$ & $2.8672 \times 10^{2}$ & $1.7054 \times 10^{-2}$ & $9.8295 \times 10^{-1}$ \\
\hline $4.7736 \times 10^{4}$ & $2.8751 \times 10^{2}$ & $1.8293 \times 10^{-2}$ & $9.8171 \times 10^{-1}$ \\
\hline $4.8993 \times 10^{4}$ & $2.8826 \times 10^{2}$ & $1.8689 \times 10^{-2}$ & $9.8131 \times 10^{-1}$ \\
\hline $5.0249 \times 10^{4}$ & $2.8900 \times 10^{2}$ & $1.8947 \times 10^{-2}$ & $9.8105 \times 10^{-1}$ \\
\hline
\end{tabular}


Table 2. Atmospheric structure for 1 bar of $\mathrm{N}_{2}$

GAM Profile

\begin{tabular}{|c|c|c|c|}
\hline $\mathrm{P}(\mathrm{Pa})$ & $\mathrm{T}(\mathrm{K})$ & $\mathrm{H} 2 \mathrm{O}$ (mass fraction) & $\mathrm{N}_{2}$ (mass fraction) \\
\hline $1.0025 \times 10^{1}$ & $2.0300 \times 10^{2}$ & $4.3187 \times 10^{-6}$ & $9.9999 \times 10^{-1}$ \\
\hline $3.0075 \times 10^{1}$ & $2.0300 \times 10^{2}$ & $4.3187 \times 10^{-6}$ & $9.9999 \times 10^{-1}$ \\
\hline $4.0100 \times 10^{1}$ & $2.0300 \times 10^{2}$ & $4.3187 \times 10^{-6}$ & $9.9999 \times 10^{-1}$ \\
\hline $5.0124 \times 10^{1}$ & $2.0300 \times 10^{2}$ & $4.3187 \times 10^{-6}$ & $9.9999 \times 10^{-1}$ \\
\hline $7.0174 \times 10^{1}$ & $2.0300 \times 10^{2}$ & $4.3187 \times 10^{-6}$ & $9.9999 \times 10^{-1}$ \\
\hline $1.0025 \times 10^{2}$ & $2.0300 \times 10^{2}$ & $4.3187 \times 10^{-6}$ & $9.9999 \times 10^{-1}$ \\
\hline $2.0050 \times 10^{2}$ & $2.0300 \times 10^{2}$ & $4.3187 \times 10^{-6}$ & $9.9999 \times 10^{-1}$ \\
\hline $3.0075 \times 10^{2}$ & $2.0300 \times 10^{2}$ & $4.3187 \times 10^{-6}$ & $9.9999 \times 10^{-1}$ \\
\hline $4.0100 \times 10^{2}$ & $2.0300 \times 10^{2}$ & $4.3187 \times 10^{-6}$ & $9.9999 \times 10^{-1}$ \\
\hline $5.0124 \times 10^{2}$ & $2.0300 \times 10^{2}$ & $4.3187 \times 10^{-6}$ & $9.9999 \times 10^{-1}$ \\
\hline $7.0174 \times 10^{2}$ & $2.0300 \times 10^{2}$ & $4.3187 \times 10^{-6}$ & $9.9999 \times 10^{-1}$ \\
\hline $1.0025 \times 10^{3}$ & $2.0300 \times 10^{2}$ & $4.3187 \times 10^{-6}$ & $9.9999 \times 10^{-1}$ \\
\hline $2.0050 \times 10^{3}$ & $2.0300 \times 10^{2}$ & $4.3187 \times 10^{-6}$ & $9.9999 \times 10^{-1}$ \\
\hline $3.0075 \times 10^{3}$ & $2.0300 \times 10^{2}$ & $4.3187 \times 10^{-6}$ & $9.9999 \times 10^{-1}$ \\
\hline $4.0100 \times 10^{3}$ & $2.0300 \times 10^{2}$ & $4.3187 \times 10^{-6}$ & $9.9999 \times 10^{-1}$ \\
\hline $5.0124 \times 10^{3}$ & $2.0300 \times 10^{2}$ & $4.3187 \times 10^{-6}$ & $9.9999 \times 10^{-1}$ \\
\hline $7.0174 \times 10^{3}$ & $2.0300 \times 10^{2}$ & $4.3187 \times 10^{-6}$ & $9.9999 \times 10^{-1}$ \\
\hline $1.0025 \times 10^{4}$ & $2.0300 \times 10^{2}$ & $4.3187 \times 10^{-6}$ & $9.9999 \times 10^{-1}$ \\
\hline $1.5037 \times 10^{4}$ & $2.0300 \times 10^{2}$ & $4.3187 \times 10^{-6}$ & $9.9999 \times 10^{-1}$ \\
\hline $2.0050 \times 10^{4}$ & $2.0300 \times 10^{2}$ & $4.3187 \times 10^{-6}$ & $9.9999 \times 10^{-1}$ \\
\hline $2.1443 \times 10^{4}$ & $2.0300 \times 10^{2}$ & $4.3187 \times 10^{-6}$ & $9.9999 \times 10^{-1}$ \\
\hline $2.5062 \times 10^{4}$ & $2.1275 \times 10^{2}$ & $1.2022 \times 10^{-5}$ & $9.9999 \times 10^{-1}$ \\
\hline $3.0075 \times 10^{4}$ & $2.2434 \times 10^{2}$ & $4.6206 \times 10^{-5}$ & $9.9995 \times 10^{-1}$ \\
\hline $3.0902 \times 10^{4}$ & $2.2600 \times 10^{2}$ & $5.9372 \times 10^{-5}$ & $9.9994 \times 10^{-1}$ \\
\hline $3.5087 \times 10^{4}$ & $2.3439 \times 10^{2}$ & $1.2202 \times 10^{-4}$ & $9.9988 \times 10^{-1}$ \\
\hline $4.0100 \times 10^{4}$ & $2.4306 \times 10^{2}$ & $2.6402 \times 10^{-4}$ & $9.9974 \times 10^{-1}$ \\
\hline $4.5112 \times 10^{4}$ & $2.5048 \times 10^{2}$ & $4.8188 \times 10^{-4}$ & $9.9952 \times 10^{-1}$ \\
\hline $5.0124 \times 10^{4}$ & $2.5677 \times 10^{2}$ & $7.7779 \times 10^{-4}$ & $9.9922 \times 10^{-1}$ \\
\hline $5.5137 \times 10^{4}$ & $2.6212 \times 10^{2}$ & $1.2086 \times 10^{-3}$ & $9.9879 \times 10^{-1}$ \\
\hline $6.0149 \times 10^{4}$ & $2.6668 \times 10^{2}$ & $1.7765 \times 10^{-3}$ & $9.9822 \times 10^{-1}$ \\
\hline $6.0554 \times 10^{4}$ & $2.6700 \times 10^{2}$ & $1.8262 \times 10^{-3}$ & $9.9817 \times 10^{-1}$ \\
\hline $6.5162 \times 10^{4}$ & $2.7063 \times 10^{2}$ & $2.3816 \times 10^{-3}$ & $9.9762 \times 10^{-1}$ \\
\hline $7.0174 \times 10^{4}$ & $2.7408 \times 10^{2}$ & $3.0298 \times 10^{-3}$ & $9.9697 \times 10^{-1}$ \\
\hline $7.2680 \times 10^{4}$ & $2.7566 \times 10^{2}$ & $3.3666 \times 10^{-3}$ & $9.9663 \times 10^{-1}$ \\
\hline $7.5187 \times 10^{4}$ & $2.7716 \times 10^{2}$ & $3.7070 \times 10^{-3}$ & $9.9629 \times 10^{-1}$ \\
\hline $7.7693 \times 10^{4}$ & $2.7859 \times 10^{2}$ & $4.0392 \times 10^{-3}$ & $9.9596 \times 10^{-1}$ \\
\hline $8.0199 \times 10^{4}$ & $2.7995 \times 10^{2}$ & $4.3754 \times 10^{-3}$ & $9.9562 \times 10^{-1}$ \\
\hline $8.0297 \times 10^{4}$ & $2.8000 \times 10^{2}$ & $4.3896 \times 10^{-3}$ & $9.9561 \times 10^{-1}$ \\
\hline $8.2705 \times 10^{4}$ & $2.8125 \times 10^{2}$ & $4.7962 \times 10^{-3}$ & $9.9520 \times 10^{-1}$ \\
\hline $8.5211 \times 10^{4}$ & $2.8249 \times 10^{2}$ & $5.3959 \times 10^{-3}$ & $9.9460 \times 10^{-1}$ \\
\hline $8.7718 \times 10^{4}$ & $2.8368 \times 10^{2}$ & $6.1941 \times 10^{-3}$ & $9.9381 \times 10^{-1}$ \\
\hline $9.0224 \times 10^{4}$ & $2.8483 \times 10^{2}$ & $7.0992 \times 10^{-3}$ & $9.9290 \times 10^{-1}$ \\
\hline $9.2730 \times 10^{4}$ & $2.8593 \times 10^{2}$ & $7.9759 \times 10^{-3}$ & $9.9202 \times 10^{-1}$ \\
\hline $9.5236 \times 10^{4}$ & $2.8699 \times 10^{2}$ & $8.7132 \times 10^{-3}$ & $9.9129 \times 10^{-1}$ \\
\hline $9.7743 \times 10^{4}$ & $2.8801 \times 10^{2}$ & $9.0575 \times 10^{-3}$ & $9.9094 \times 10^{-1}$ \\
\hline $1.0025 \times 10^{5}$ & $2.8900 \times 10^{2}$ & $9.3308 \times 10^{-3}$ & $9.9067 \times 10^{-1}$ \\
\hline
\end{tabular}


Table 3. Atmospheric structure for 2 bar of $\mathrm{N}_{2}$

GAM Profile

\begin{tabular}{|c|c|c|c|}
\hline $\mathrm{P}(\mathrm{Pa})$ & $\mathrm{T}(\mathrm{K})$ & $\mathrm{H} 2 \mathrm{O}$ (mass fraction) & $\mathrm{N}_{2}$ (mass fraction) \\
\hline $2.0025 \times 10^{1}$ & $2.0300 \times 10^{2}$ & $1.6596 \times 10^{-6}$ & $9.9999 \times 10^{-1}$ \\
\hline $6.0075 \times 10^{1}$ & $2.0300 \times 10^{2}$ & $1.6596 \times 10^{-6}$ & $9.9999 \times 10^{-1}$ \\
\hline $8.0100 \times 10^{1}$ & $2.0300 \times 10^{2}$ & $1.6596 \times 10^{-6}$ & $9.9999 \times 10^{-1}$ \\
\hline $1.0012 \times 10^{2}$ & $2.0300 \times 10^{2}$ & $1.6596 \times 10^{-6}$ & $9.9999 \times 10^{-1}$ \\
\hline $1.4017 \times 10^{2}$ & $2.0300 \times 10^{2}$ & $1.6596 \times 10^{-6}$ & $9.9999 \times 10^{-1}$ \\
\hline $2.0025 \times 10^{2}$ & $2.0300 \times 10^{2}$ & $1.6596 \times 10^{-6}$ & $9.9999 \times 10^{-1}$ \\
\hline $4.0050 \times 10^{2}$ & $2.0300 \times 10^{2}$ & $1.6596 \times 10^{-6}$ & $9.9999 \times 10^{-1}$ \\
\hline $6.0075 \times 10^{2}$ & $2.0300 \times 10^{2}$ & $1.6596 \times 10^{-6}$ & $9.9999 \times 10^{-1}$ \\
\hline $8.0100 \times 10^{2}$ & $2.0300 \times 10^{2}$ & $1.6596 \times 10^{-6}$ & $9.9999 \times 10^{-1}$ \\
\hline $1.0012 \times 10^{3}$ & $2.0300 \times 10^{2}$ & $1.6596 \times 10^{-6}$ & $9.9999 \times 10^{-1}$ \\
\hline $1.4017 \times 10^{3}$ & $2.0300 \times 10^{2}$ & $1.6596 \times 10^{-6}$ & $9.9999 \times 10^{-1}$ \\
\hline $2.0025 \times 10^{3}$ & $2.0300 \times 10^{2}$ & $1.6596 \times 10^{-6}$ & $9.9999 \times 10^{-1}$ \\
\hline $4.0050 \times 10^{3}$ & $2.0300 \times 10^{2}$ & $1.6596 \times 10^{-6}$ & $9.9999 \times 10^{-1}$ \\
\hline $6.0075 \times 10^{3}$ & $2.0300 \times 10^{2}$ & $1.6596 \times 10^{-6}$ & $9.9999 \times 10^{-1}$ \\
\hline $8.0100 \times 10^{3}$ & $2.0300 \times 10^{2}$ & $1.6596 \times 10^{-6}$ & $9.9999 \times 10^{-1}$ \\
\hline $1.0012 \times 10^{4}$ & $2.0300 \times 10^{2}$ & $1.6596 \times 10^{-6}$ & $9.9999 \times 10^{-1}$ \\
\hline $1.4017 \times 10^{4}$ & $2.0300 \times 10^{2}$ & $1.6596 \times 10^{-6}$ & $9.9999 \times 10^{-1}$ \\
\hline $2.0025 \times 10^{4}$ & $2.0300 \times 10^{2}$ & $1.6596 \times 10^{-6}$ & $9.9999 \times 10^{-1}$ \\
\hline $3.0037 \times 10^{4}$ & $2.0300 \times 10^{2}$ & $1.6596 \times 10^{-6}$ & $9.9999 \times 10^{-1}$ \\
\hline $4.0050 \times 10^{4}$ & $2.0300 \times 10^{2}$ & $1.6596 \times 10^{-6}$ & $9.9999 \times 10^{-1}$ \\
\hline $5.0062 \times 10^{4}$ & $2.0300 \times 10^{2}$ & $1.6596 \times 10^{-6}$ & $9.9999 \times 10^{-1}$ \\
\hline $5.0885 \times 10^{4}$ & $2.0300 \times 10^{2}$ & $1.6596 \times 10^{-6}$ & $9.9999 \times 10^{-1}$ \\
\hline $6.0075 \times 10^{4}$ & $2.1323 \times 10^{2}$ & $5.5496 \times 10^{-6}$ & $9.9999 \times 10^{-1}$ \\
\hline $7.0087 \times 10^{4}$ & $2.2310 \times 10^{2}$ & $1.6186 \times 10^{-5}$ & $9.9998 \times 10^{-1}$ \\
\hline $7.3373 \times 10^{4}$ & $2.2600 \times 10^{2}$ & $2.3852 \times 10^{-5}$ & $9.9998 \times 10^{-1}$ \\
\hline $8.0100 \times 10^{4}$ & $2.3194 \times 10^{2}$ & $3.9254 \times 10^{-5}$ & $9.9996 \times 10^{-1}$ \\
\hline $9.0112 \times 10^{4}$ & $2.3991 \times 10^{2}$ & $8.1710 \times 10^{-5}$ & $9.9992 \times 10^{-1}$ \\
\hline $1.0012 \times 10^{5}$ & $2.4711 \times 10^{2}$ & $1.5223 \times 10^{-4}$ & $9.9985 \times 10^{-1}$ \\
\hline $1.1014 \times 10^{5}$ & $2.5357 \times 10^{2}$ & $2.7372 \times 10^{-4}$ & $9.9973 \times 10^{-1}$ \\
\hline $1.2015 \times 10^{5}$ & $2.5937 \times 10^{2}$ & $4.6244 \times 10^{-4}$ & $9.9954 \times 10^{-1}$ \\
\hline $1.3016 \times 10^{5}$ & $2.6455 \times 10^{2}$ & $7.0383 \times 10^{-4}$ & $9.9930 \times 10^{-1}$ \\
\hline $1.3548 \times 10^{5}$ & $2.6700 \times 10^{2}$ & $8.6121 \times 10^{-4}$ & $9.9914 \times 10^{-1}$ \\
\hline $1.4017 \times 10^{5}$ & $2.6917 \times 10^{2}$ & $1.0103 \times 10^{-3}$ & $9.9899 \times 10^{-1}$ \\
\hline $1.4518 \times 10^{5}$ & $2.7129 \times 10^{2}$ & $1.1975 \times 10^{-3}$ & $9.9880 \times 10^{-1}$ \\
\hline $1.5019 \times 10^{5}$ & $2.7330 \times 10^{2}$ & $1.3995 \times 10^{-3}$ & $9.9860 \times 10^{-1}$ \\
\hline $1.5519 \times 10^{5}$ & $2.7520 \times 10^{2}$ & $1.5814 \times 10^{-3}$ & $9.9842 \times 10^{-1}$ \\
\hline $1.6020 \times 10^{5}$ & $2.7702 \times 10^{2}$ & $1.7737 \times 10^{-3}$ & $9.9823 \times 10^{-1}$ \\
\hline $1.6521 \times 10^{5}$ & $2.7875 \times 10^{2}$ & $2.0086 \times 10^{-3}$ & $9.9799 \times 10^{-1}$ \\
\hline $1.6898 \times 10^{5}$ & $2.8000 \times 10^{2}$ & $2.2395 \times 10^{-3}$ & $9.9776 \times 10^{-1}$ \\
\hline $1.7021 \times 10^{5}$ & $2.8041 \times 10^{2}$ & $2.3270 \times 10^{-3}$ & $9.9767 \times 10^{-1}$ \\
\hline $1.7522 \times 10^{5}$ & $2.8199 \times 10^{2}$ & $2.7457 \times 10^{-3}$ & $9.9725 \times 10^{-1}$ \\
\hline $1.8022 \times 10^{5}$ & $2.8351 \times 10^{2}$ & $3.2299 \times 10^{-3}$ & $9.9677 \times 10^{-1}$ \\
\hline $1.8523 \times 10^{5}$ & $2.8496 \times 10^{2}$ & $3.7165 \times 10^{-3}$ & $9.9628 \times 10^{-1}$ \\
\hline $1.9024 \times 10^{5}$ & $2.8636 \times 10^{2}$ & $4.1525 \times 10^{-3}$ & $9.9585 \times 10^{-1}$ \\
\hline $1.9524 \times 10^{5}$ & $2.8771 \times 10^{2}$ & $4.4087 \times 10^{-3}$ & $9.9559 \times 10^{-1}$ \\
\hline $2.0025 \times 10^{5}$ & $2.8900 \times 10^{2}$ & $4.6306 \times 10^{-3}$ & $9.9537 \times 10^{-1}$ \\
\hline
\end{tabular}

